\title{
The Effect of High Strain Rate on the Piezoresistance of Polydimethylsiloxane with Carbon Nanotubes
}

\author{
Farouk Wahsh \\ Mechanical Engineering Department \\ York University \\ Toronto, Canada \\ faroukw@my.yorku.ca
}

\author{
Aleksander Czekanski \\ Mechanical Engineering Department \\ York University \\ Toronto, Canada \\ alex.czekanski@lassonde.yorku.ca
}

\begin{abstract}
This paper describes the piezoresistive properties of a nanocomposite made of polydimethylsiloxane and multiwalled carbon nanotubes that is subjected to high strain rate. We highlight the production process of the composite, the testing protocol, and the results obtained.
\end{abstract}

Keywords: piezoresistance; nanocomposite; high strain rate; polydimethylsiloxane; carbon nanotubes; Kolsky bar

\section{INTRODUCTION}

Over the past decade, smart materials have become increasingly popular owing to their intrinsic functional properties that allow for fast, easy, and inexpensive sensing and actuating.

Composites based on nanoscale fillers such as carbon nanotubes (CNTs) are considered the new generation of smart materials. Since their discovery in 1991, CNTs have had a significant impact on the field of material science. They are cylindrical in shape and consist of carbon molecules covalently bonded together to form a tube-shaped lattice, lending them their unique properties - superior electrical and magnetic conductivity, lightness, and strength. Polydimethylsiloxane (PDMS) is the simplest member of the silicone polymer family and is characterized by its attractive mechanical properties and translucency.

Much work has been done on developing highly sensitive strain sensors made from CNT-polymer nanocomposites. This paper investigates the potential of PDMS mixed with CNTs in the development of high strain rate (impact) sensors.

\section{MethodOLOGY}

\section{A. Composite Production Process}

The material in question is an elastomeric composite made from PDMS as the matrix and multi-walled carbon nanotubes (MWCNTs) as the phase. The production of this composite was a complex, time-consuming, and dangerous process. First, there is the issue of avoiding exposure, since CNTs are considered hazardous and not enough research has been done on the risks they pose to human health. Second, the dispersion of CNTs in PDMS was difficult to achieve. The reason for that is the tendency of CNTs to agglomerate over time when mixed in liquids. To prevent these agglomerations, chloroform was used as a solvent to aid the dispersion [1], and the material was sonicated repeatedly throughout the process. Third, the curing time of PDMS at room temperature is unfavorably long at 48 to $72 \mathrm{~h}$. This creates a wide window in which CNTs can agglomerate. To remedy this problem, the PDMS was cured at $110^{\circ} \mathrm{C}$, which reduced curing time to $30 \mathrm{~min}$. Last, to cure the material at high temperature, a custom press-casting heated mold was developed.

The mold was made from two blocks of aluminum cut using computer numerical control (CNC). The bottom part (drag) contained the PDMS, while the top part (cope) had an emboss that forced the PDMS on the surface out of the cast area and into channels on all four sides of the mold that contained the excess material. The cope was hinged to the drag of the mold and closed with a mechanism resembling that of a waffle maker. This design prevents imperfections caused by air pockets and bubbles that would otherwise get trapped during assembly below the cope and inside the cast. Moreover, three though-all holes were drilled in the cope below the surface of the cast to house two cartridge heaters and one thermocouple. The heaters were connected to a relay, a thermocouple, and a PID controller in a circuit that controls the temperature inside the mold according to the user's selection.

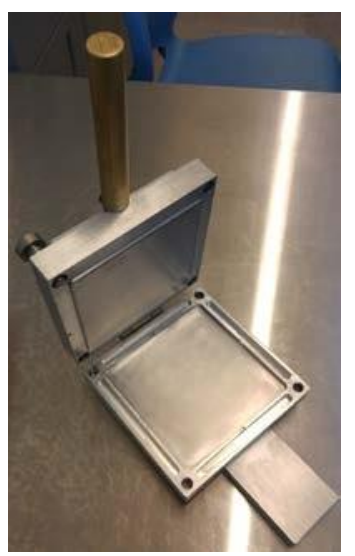

Figure 1. Heated Press-casting Mold 


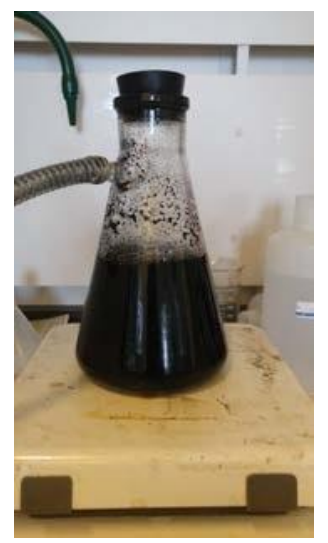

Figure 2. PDMS/CNT Solution - Chloroform Expulsion

Our primary objective was to study the effect of CNT concentration in PDMS on the electrical resistivity when the material was subjected to high strain rates. Therefore, three different CNT concentrations were studied, ranging from $1 \mathrm{wt}$. $\%$ to 2 wt. $\%$, with 0.5 wt. $\%$ increments.

Dispersion is a spatial quality that represents how evenly distributed CNTs are in the PDMS matrix. Manually mixing CNTs in PDMS does not generate enough shear forces to overcome the inter-tube Van der Waals forces and separate the tubes from their aggregations [1]. On the other hand, more advanced techniques such as mechanical stirring and sonication were found to deliver better results in dispersing nanoparticles in solutions. However, the efficiency of these methods is greatly reduced with the use of highly viscous liquids such as PDMS. Therefore, a solvent was used to dissolve the PDMS, thereby reducing its viscosity. Chloroform was chosen for two reasons. One, CNTs were found to disperse well in chloroform compared with their dispersion in other reagents like toluene [1]. Two, chloroform dissolved PDMS without chemically reacting with it, unlike other reagents that might provide better dispersibility. Once the dispersion process was complete, the solution was heated to evaporate the chloroform and expel it from the solution. To speed up the process, a vacuum was introduced to encourage the chloroform's evaporation as shown in Fig. 2. Finally, the solution was cast in the mold to produce a $3 \mathrm{~mm} \times 120 \mathrm{~mm} \times 120 \mathrm{~mm}$ sheet of the composite.

The production process ${ }^{1}$ of the material is summarized in the following eight steps:

1. Chloroform was mixed with MWCNTs at a ratio of 1/15 g.mL $\mathrm{mL}^{-1}$ (MWCNT: $\mathrm{CHCl}_{3}$ ) in a beaker and sonicated for $1 \mathrm{~h}$.

2. Chloroform was mixed with PDMS part A at a ratio of

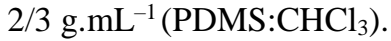

3. The solutions from steps 1 and 2 were mixed and sonicated for $3 \mathrm{~h}$.

4. The solution from step 3 was heated in an Erlenmeyer flask under vacuum at $62{ }^{\circ} \mathrm{C}$ while mechanically stirring at $1200 \mathrm{r} . \mathrm{min}^{-1}$ for $3 \mathrm{~h}$ to expel the chloroform.

${ }^{1}$ Applicable to 1 wt. $\%$ of CNTs. For $1.5 \mathrm{wt} . \%$ and 2 wt. \%, the concentration of CNTs in PDMS was adjusted accordingly.

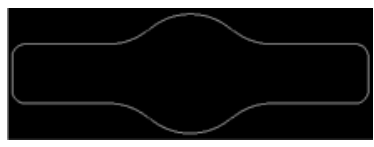

Figure 3. Sample Shape

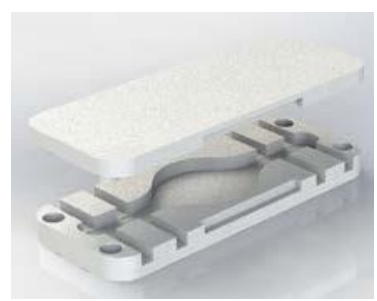

Figure 4. Probe Insertion Guide Housing

5. Part B was added to the solution from step 4, and this solution was manually mixed for $10 \mathrm{~min}$.

6. The solution from step 5 was put in a vacuum chamber to remove any air bubbles trapped under the surface.

7. After degassing, the solution was carefully cast in the mold at a controlled temperature of $110^{\circ} \mathrm{C}$ for $30 \mathrm{~min}$.

8. The mold was carefully opened, and the sheet casting was removed from it.

To prepare the material for testing, samples were laser cut from the sheets produced using an Epilog Laser Fusion M2 cutter in the shape shown in Fig. 3.

To measure the electrical resistance of the material, a fourwire connection [2] was used to control current through the sample and measure the voltage change along the sample. The distance between the probes was controlled for all samples, since it impacts measured potential significantly. A fixed distance was achieved by the design and fused deposition modelling of a small housing for the samples that guide the insertion of the probes, as shown in Fig. 4.

\section{B. Testing}

Testing the material's electrical resistance response to high strain rate was performed using a Kolsky (Split-Hopkinson) (Fig. 5). The Kolsky bar was manufactured in house [3] and was used to subject the material to high strain rates. MATLAB was used for system control, and a National Instruments USB6351 DAQ device was used for data acquisition.

After the probes were inserted in the sample and connected to current and voltage source meters following the four-wire connection, the sample was sandwiched between the incident and transmitted bars of the Kolsky bar, as shown in Fig. 4.

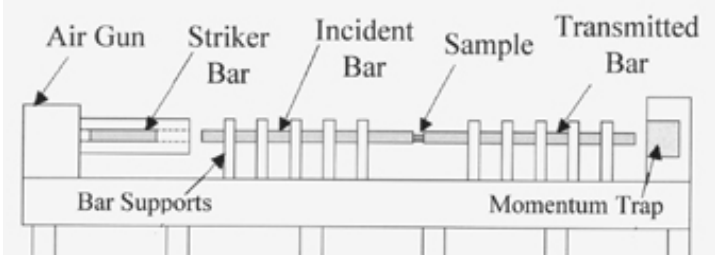

Figure 5. Kolsky (Split-Hopkinson) Bar Schematic 


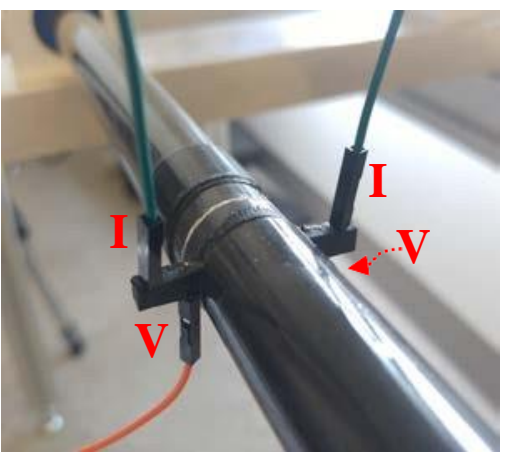

Figure 6. Sample Setup on Kolsky Apparatus

The air-gun pressure was kept constant at 100 psi for all tests to approach reproducibility of results. The DAQ was synced with the trigger through a MATLAB code such that it started collecting data from the strain gauges and the sample the moment the trigger on the Kolsky bar was pulled. The data sampling went on for $5 \mathrm{~s}$ from the moment the trigger was pulled.

\section{RESULTS AND OBSERVATIONS}

\section{A. Composite Production}

The sheets of composite produced were checked for quality to ensure samples were as uniform as possible. First, the surface of the sheet was inspected for cavities caused by trapped air. Second, the sheets were cut in different areas, and the cross-section was inspected for cavities as well. Since the material is black, imperfections within each sample were hard to detect nondestructively. However, a method used to test the uniformity of the sample ensured that the initial resistance of the samples was within the same range. In that way, samples with significant imperfections were eliminated.

We faced several challenges during the production process. First, the chloroform had to be completely evaporated from the material before it was cast. Any remaining chloroform would threaten the chemical purity of the composite, leading to incorrect results. Excess chloroform also affects the physical structure of the final composite. If chloroform exists in the material casted, it will quickly evaporate inside the mold because of the high curing temperature. This creates cavities at the surface of the casting and affects its physical structure, as shown in Fig. 7(a).

Achieving good results depended on trial and error. It took three attempts to finally arrive at a reliable process that produced good-quality sheets of PDMS with CNTs. The sheets produced were free from surface and body imperfections according to our visual inspections. Fig. 7(b) shows a successfully produced sample. Samples were laser cut from these sheets for testing.
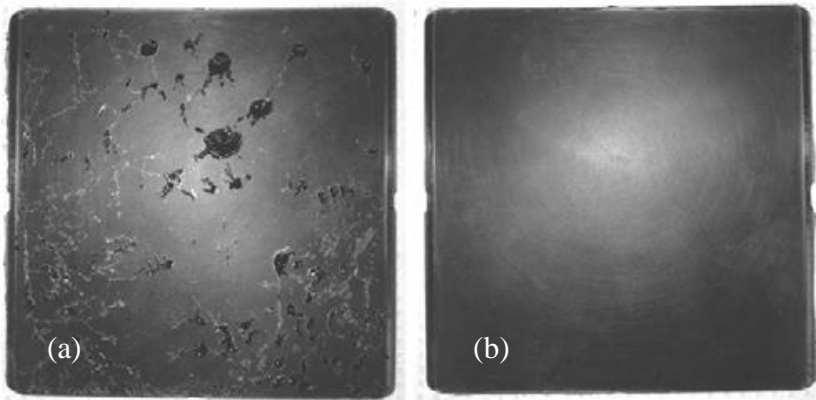

Figure 7. (a) Incomplete Chloroform Evaporation. (b) Complete Chloroform Evaporation.

\section{B. Test Results and Observations}

Dynamic tests were conducted on five samples for each concentration of CNTs in PDMS. The results obtained were found to be consistent and exhibited similar trends. However, only data from one representative set of samples are presented here.

Fig. 8 shows the voltage measured from the sample during the initial moments of impact. The normalized voltage from the strain gauges on the incident and transmitted bars is also shown and represents the stress waves propagating through the system. Early observation of the CNT-PDMS plot shows a spike in voltage reaching up to 2.5 times the initial voltage. Moreover, the voltage was found to rise in steps that are synced with the propagating stress waves in the system. Furthermore, the sample voltage was observed to lag half a period behind the incident wave and was in phase with the transmitted wave.

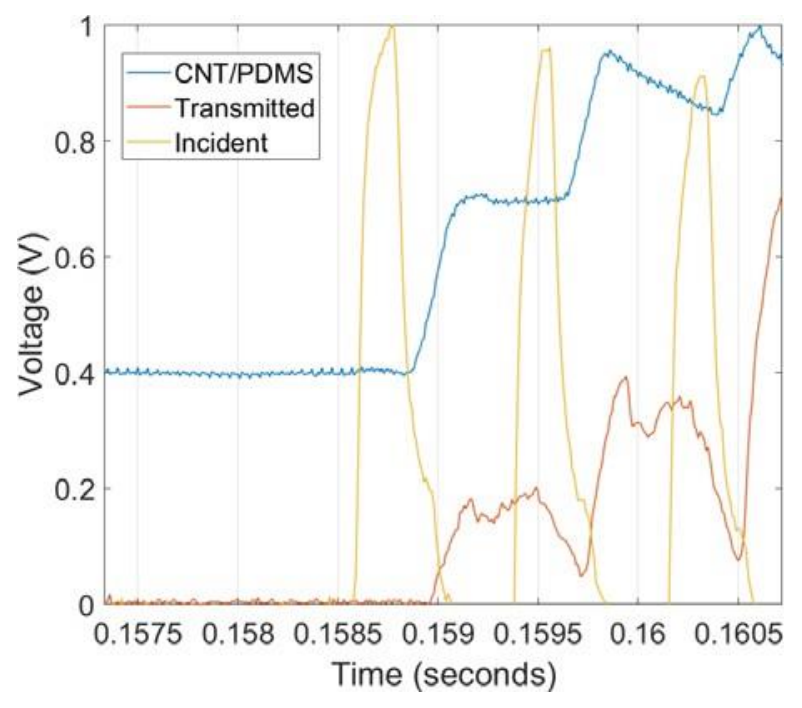

Figure 8. Transmitted and incident voltage output. (1wt. \% sample)

Fig. 9 shows the resistance change within the sample plotted against the strain. The stress-strain curve of the material is also plotted on the graph to emphasize the observed correlation. The change in resistance aligned with the stress/strain curve of the sample, indicating a strong correlation between electrical resistance and applied stress on the material. 


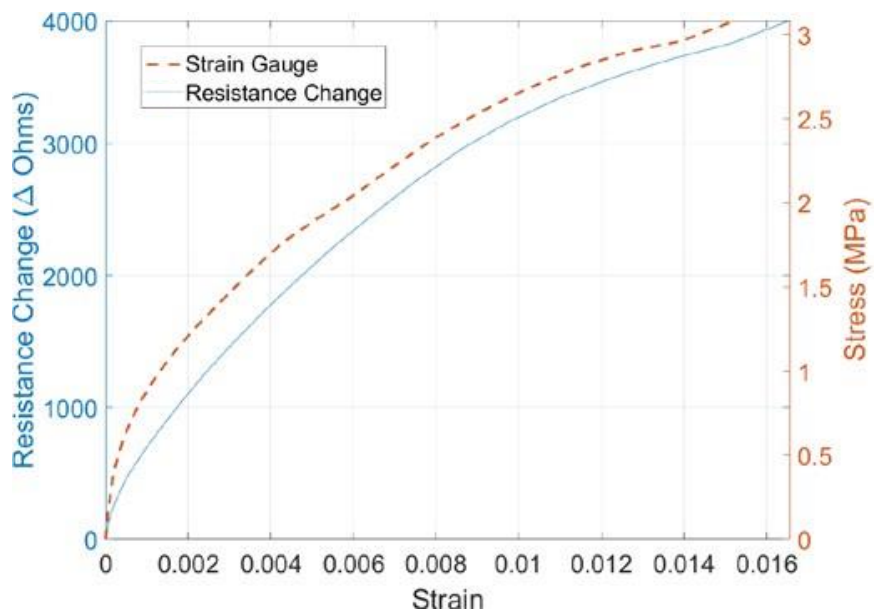

Figure 9. Strain Gauge and Resistance Change vs. Strain at Initial Contact

Fig. 10 shows the change in resistance within samples of different CNT concentration plotted against time. The observed rise in resistance was consistent at all three concentrations. However, we observed that increasing the CNT concentration in samples led to smaller resistance changes within them. The rate at which the resistance of the composite changed decreased when CNT concentration in the PDMS increased.

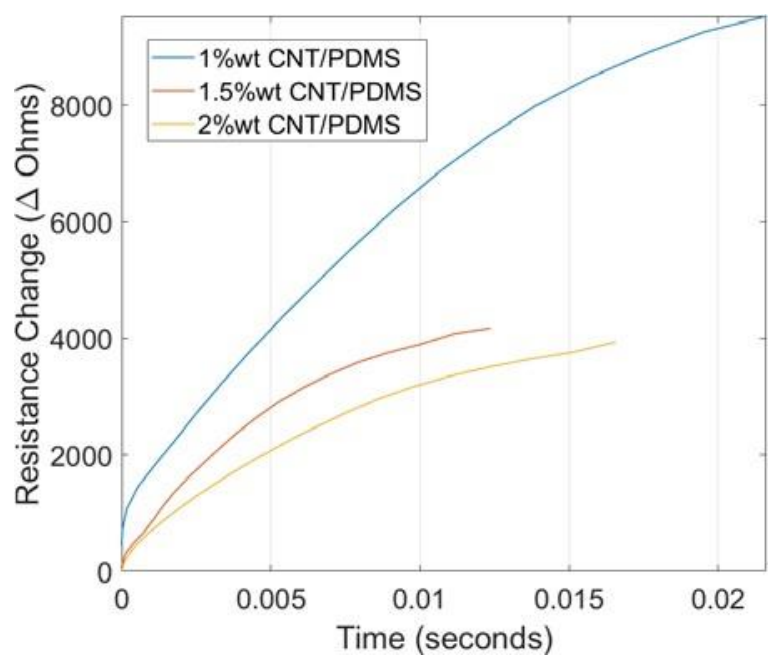

Figure 10. Resistance Change with respect to time for different CNT/PDMS weight percentage.

\section{CONCLUSION}

The effect of high strain rate on the piezoresistive properties of elastomeric composites shows promise in the potential development of high strain rate sensors. The results show a strong correlation between high strain rate and electrical resistance, indicating piezoresistive behavior in the material.

The elastomeric composite was prepared using Sylgard 184 PDMS and MWCNTs. The material was sonicated to break down the CNT chains and agglomerations. Our results show that chloroform functioned well as a solvent for the purposes of dispersing CNTs in PDMS. A custom press casting mold was developed to efficiently cast sheets of the material that were then laser cut to produce the samples for dynamic testing using the Kolsky bar. During tests, the current through the material was controlled, and the voltage and strain were measured.

The electrical resistance was found to spike upon loading with a lag of half a period relative to the incident wave and inphase relative to the transmitted wave. The slow recovery of voltage indicates viscoelastic behavior in the material [4]. The magnitude of the peak resistance was found to be 2.5 times the initial resistance, which represents the signal to be detected. The peak value was found to be directly related to the strain rate (as further research will show). By detecting this peak, we hope to determine the strain rate through means of statistical analysis. This research has proven the potential of CNTimpregnated elastomers for the development of impact force sensors. Future work includes further testing on the material to confirm preliminary results and gather enough data to build a robust statistical model. The goal of the model is to generate stress values from the input, the measured electrical resistance across the material.

\section{ACKNOWLEDGMENT}

The authors gratefully acknowledge the generous financial support of Lassonde School of Engineering (York University) and NSERC-Quanser Chair in Design Engineering.

\section{REFERENCES}

[1] Liu, C., \& Choi, J. (2012). "Improved Dispersion of Carbon Nanotubes in Polymers at High Concentrations. Nanomaterials", 2(4), 329-347. http://dx.doi.org/10.3390/nano2040329

[2] Heaney, Michael. (2003). Electrical Conductivity and Resistivity. 7-1 to 7. Electrical Measurement, Signal Processing, and Displays. Ed. John G.Webster. CRC Press.

[3] Chaudhry, M. S. (2017). On the Characterization of Engineered Elastomers At High Strain Rates(Unpublished master's thesis). York University. Retrieved August 05, 2017, from https://yorkspace.library.yorku.ca/xmlui/handle/10315/33410

[4] Zezin, Y., \& Lomakin, E. (2015). Study of Viscoelastic Properties of Elastomers Reinforced by Nanoparticles. Mechanics Of Solids, 50(2), 117-126. http://dx.doi.org/10.3103/s0025654415020016 